\title{
BRIP1, a Gene Potentially Implicated in Familial Colorectal Cancer Type $\mathbf{X}$
}

\author{
Lorena Martín-Morales', Pilar Garre', Víctor Lorca', Marta Cazorla', Patricia Llovet', \\ Inmaculada Bando', Vanesa García-Barberan', María Luisa González-Morales², \\ Clara Esteban-Jurado ${ }^{3}$, Miguel de la Hoya', Sergi Castellví-Bel ${ }^{3}$, and Trinidad Caldés ${ }^{1}$
}

\section{ABSTRACT}

Familial colorectal cancer Type X (FCCTX) comprises a heterogeneous group of families with an increased risk of developing colorectal cancer and other related tumors, but with mismatch repair-proficient, microsatellite-stable (MSS) tumors. Unfortunately, the genetic basis underlying their cancer predisposition remains unknown. Although pathogenic germline variants in BRIP1 increase the risk of developing hereditary ovarian cancer, the involvement of BRIP1 in hereditary colorectal cancer is still not well known. In order to identify new BRIP1 variants associated with inherited colorectal cancer, affected and nonaffected individuals from 18 FCCTX or high-risk MSS colorectal cancer families were evaluated by whole-exome sequencing, and another 62 colorectal cancer patients from FCCTX or high-risk MSS colorectal cancer families were screened by a next-generation sequencing (NGS) multigene panel. The families were recruited at the Genetic Counseling Unit of Hospital Clínico San Carlos of Madrid. A total of three different BRIP1 mutations in three unrelated families were identified. Among them, there were

\section{Introduction}

Colorectal cancer is the third most common cancer and the second leading cancer-related cause of death in the world $(1,2)$. It is estimated that familial risk is involved in up to $30 \%$ of all colorectal cancer cases $(3,4)$, although not more than $5 \%$ to $6 \%$ are caused by known germline mutations in cancer-

'Molecular Oncology Laboratory, Hospital Clínico San Carlos, IdISSC, Centro de Investigación Biomédica en Red de Oncología (CIBERONC), Madrid, Spain ${ }^{2}$ Department of Pathology, Hospital Clínico San Carlos, IdISSC, Madrid, Spain. ${ }^{3}$ Gastroenterology Department, Hospital Clínic, Institut d'Investigacions Biomèdiques August Pi i Sunyer (IDIBAPS), Centro de Investigación Biomédica en Red de Enfermedades Hepáticas y Digestivas (CIBEREHD), Barcelona, Spain.

Note: Supplementary data for this article are available at Cancer Prevention Research Online (http://cancerprevres.aacrjournals.org/).

L. Martín-Morales and P. Garre contributed equally to this work.

Corresponding Author: Trinidad Caldés, Molecular Oncology Laboratory, Hospital Clínico San Carlos, IdISSC, Martin Lagos s/n, Madrid 28040, Spain. Phone: 34-91330-3348; Fax: 34-91330-3544; E-mail:

trinidad.caldes@salud.madrid.org

Cancer Prev Res 2021;14:185-94

doi: 10.1158/1940-6207.CAPR-20-0316

(C)2020 American Association for Cancer Research. two frameshift variants [c.1702_1703del, p.(Asn568TrpfsTer9) and c.903del, p.(Leu301PhefsTer2)] that result in the truncation of the protein and are thus classified as pathogenic (class 5). The remaining was a missense variant $[c .2220 \mathrm{G}>\mathrm{T}$, p.(Gln740His)] considered a variant of uncertain significance (class 3 ). The segregation and lossof-heterozygosity studies provide evidence linking the two BRIP1 frameshift variants to colorectal cancer risk, with suggestive but not definitive evidence that the third variant may be benign. The results here presented suggest that germline BRIP1 pathogenic variants could be associated with hereditary colorectal cancer predisposition.

Prevention Relevance: We suggest that BRIP1 pathogenic germline variants may have a causal role in CRC as moderate cancer susceptibility alleles and be associated with hereditary CRC predisposition. A better understanding of hereditary CRC may provide important clues to disease predisposition and could contribute to molecular diagnostics, improved risk stratification, and targeted therapeutic strategies.

predisposing genes (5). The most common form of inherited colorectal cancer is hereditary nonpolyposis colorectal cancer (HNPCC), a familial syndrome characterized by an increased incidence of colorectal cancer and other tumors (6) that is defined by the Amsterdam I and II clinical criteria $(7,8)$. However, only half of these families are explained by germline inactivating mutations in the mismatch repair (MMR) genes. These cases, known as Lynch syndrome, present tumors that lack the corresponding MMR proteins and fail to repair DNA through this pathway, which leads to microsatellite instability (MSI) and the accumulation of somatic mutations (9). Although HNPCC and Lynch syndrome have been used as synonyms in the past, nowadays HNPCC is defined by the clinical criteria, whereas Lynch syndrome refers to those families with germline MMR defects (10). On the other hand, the Bethesda guidelines were designed to select those high-risk colorectal cancer families that should undergo MMR testing (11).

The other half of HNPCC families are MMR proficient and present microsatellite-stable (MSS) tumors. These cases have been grouped under the term familial colorectal cancer type $\mathrm{X}$ (FCCTX), and the genetic basis underlying their cancer predisposition remains unknown (12). Several studies have 
reported that FCCTX tumors show different molecular and clinical features than both Lynch syndrome and sporadic colorectal cancer, as well as the deregulation of different canonical pathways, such as the Wnt, EGFR, and p53 pathways (13-15). Nonetheless, FCCTX is a heterogeneous group of families, and we are still far from fully understanding the different events and genetic alterations involved in their tumor progression and heredity. Although previous studies had identified a few genes involved in the cancer susceptibility of these families $(16,17)$, it has not been until the arrival of nextgeneration sequencing (NGS) that a larger amount of cancerpredisposing genes are being discovered (18-23). According to published results, it seems that FCCTX does not form a single entity, because multiple different genes are involved in their cancer heritability. However, altogether these still explain the inheritance in only a small portion of the families. Thus, the identification of new high-risk genes that contribute to the increased cancer susceptibility of FCCTX families is still a challenge and a priority.

In a previous collaborative study between our hospital (Hospital Clínico San Carlos of Madrid) and the Epicolon consortium at Hospital Clínic of Barcelona, new genes that confer high risk of developing cancer were described (19). Among them, we identified one family carrying a variant in BRIP1 (BRCA1 Interacting Protein C-terminal helicase 1; ref. 24). BRIP1, also known as FANCJ/BACH1, is one of the genes linked to Fanconi anemia (FA), a genetically heterogeneous disease to which pathogenic variants in at least 22 genes have been currently associated. Eight FA complementation group proteins and three non-FA proteins form the FA core complex that is required for the monoubiquitination of the FANCD2-FANCI dimer upon DNA damage $(25,26)$. BRIP1, BRCA2, and PALB2 act in parallel or downstream of FANCD2, working collectively in a common pathway that has been termed the FA-BRCA pathway, and BRIP1's helicase domains suggest its direct involvement at DNA-repair sites (27).

Mutations in BRIP1 are observed in patients who belong to the FA-J complementation group, which is why BRIP1 is also known as FANCJ (28-30). Pathogenic BRIP1 germline mutations are also known to confer about a $10 \%$ cumulative risk of developing ovarian cancer $(31,32)$, whereas the role of $B R I P 1$ in breast cancer remains controversial $(33,34)$. On the other hand, the association between BRIP1 mutations and colorectal cancer was reported for the first time in our previous collaborative study $(19,24)$, but more recently other studies have confirmed this association (35-37).

Our group performed NGS analysis of individuals from FCCTX or other high-risk MSS colorectal cancer families without mutations in genes previously associated with colorectal cancer. Due to recent publications about BRIP1 and colorectal cancer, together with the well-known association between $B R I P 1$ and hereditary ovarian cancer $(31,38)$, the aim of this work was to evaluate the role of BRIP1 variants in inherited colorectal cancer. Here we describe three germline $B R I P 1$ variants detected in three unrelated families.

\section{Materials and Methods Study population}

Affected and nonaffected individuals from 18 FCCTX or highrisk MSS colorectal cancer families were studied by whole-exome sequencing, and another 62 colorectal cancer patients from either FCCTX or high-risk MSS families were screened by an NGS multigene panel. The families were collected at the Genetic Counseling Unit of Hospital Clínico San Carlos of Madrid. All the families fulfilled either the Amsterdam I/II criteria or the Bethesda guidelines for $\operatorname{HNPCC}(7,8,11)$. In addition, all the colorectal cancer tumors from these families were MSS and presented normal expression of the MMR proteins. The study was approved by the Institutional Review Boards of the Hospital Clínico San Carlos, and a written informed consent was obtained from each participant. Personal and family histories were obtained from the proband and participating relatives, and cancer diagnoses were confirmed by medical and pathology records.

\section{DNA extraction}

Germline DNA was extracted from peripheral blood using the MagNA Pure Compaq extractor system (Roche Diagnostics), according to the manufacturer's protocol. Tumor DNA was extracted from $7-\mu \mathrm{m}$-thick formalin-fixed paraffin-embedded (FFPE) tissue sections using the QIAamp DNA FFPE Tissue Kit (Qiagen) according to its protocol. A hematoxylin/eosin-stained section of each block allowed the assessment of tumor cell area and content by two experienced pathologists. Finally, DNA quantity and quality were evaluated using a NanoDrop (ND1000) spectrophotometer, Qubit V3.0 Fluorometer (Thermo Fisher Scientific), and agarose gel electrophoresis (concentration between 50 and $200 \mathrm{ng} / \mu \mathrm{L}$ and purity OD260/280 = 1.8-2.0).

\section{Whole-exome sequencing}

The whole-exome sequencing library was prepared according to Agilent's SureSelect protocol for Illumina paired-end sequencing (SureSelectXT Human All Exon V3, 51Mb, Agilent Technologies). The final library size and concentration were determined on an Agilent 2100 Bioanalyzer and a Qubit Fluorometer (Thermo Fisher Scientific), respectively. Finally, the library was sequenced on an Illumina HiSeq 2000 platform with paired-end reads of $250 \mathrm{bp}$, following the manufacturer's protocol. Images generated by the HiSeq 2000 were processed using the manufacturer's software to generate FASTQ sequence files. Reads were aligned against the human reference genome version GRCh37/hg19 using the BWA software, creating the BAM files. Low quality reads, PCR duplicates, and other sequences that could introduce major biases were removed using Picard-tools and SAMtools. Variant calling was performed using a combination of two different algorithms (VarScan and GATK) and the identified variants were annotated and named using the HGMD and Ensembl databases.

\section{TruSight cancer panel sequencing}

Sixty-two colorectal cancer patients were tested with the TruSight Cancer Panel, which targets 94 genes known to play 
a role in cancer predisposition. The integrated sample preparation was done following the Nextera enrichment protocol from Illumina and as little as $50 \mathrm{ng}$ of DNA for the library. Sequencing was performed on a MiSeq platform (Illumina), and data were analyzed by the MiSeq Reporter Software.

\section{Variant filtering}

The variants identified by NGS were subsequently filtered to select those variants that were (1) shared by the affected members sequenced from each family (when applicable); (2) carried in heterozygosis; (3) coding, nonsynonymous (missense, stop gain, stop loss, inframe, frameshift, and splicing) and located in autosomes; (4) rare (minor allele frequency, MAF, in the general population $\leq 0.01$ ); (5) predicted to be damaging (for missense and inframe variants) or to affect the splicing (for splice region variants) by in silico tools; and (6) not carried by elderly healthy relatives sequenced (when applicable).

\section{In silico studies}

The MAF of each variant was checked in three different databases (1000 Genomes Project, EVS, and gnomAD). On the other hand, for every missense variant, the PolyPhen, SIFT, and MutationTaster online tools were used to predict the impact of each amino acid substitution on the protein. Splice region variants were also analyzed by the Human Splicing Finder in order to predict splicing alterations.

\section{Variant validation, segregation, and loss-of- heterozygosity studies}

BRIP1 variants were validated, and their segregation was studied by PCR followed by Sanger sequencing of the corresponding region of the gene (exons 7,12 , and 15; transcript ENST00000259008.6) using specific primers designed with Primer3 (Supplementary Table S1). The segregation study was carried out in germline DNA from the available members of each family. However, although no germline DNA was available from the deceased member II:1 of family CC41 (Fig. 1), we were able to study the segregation in this member using tumor DNA. The loss-of-heterozygosity ( $\mathrm{LOH}$ ) was assessed in tumor DNA by either Sanger sequencing or the study of microsatellites. For this purpose, germline and tumor DNA were compared, allowing the discrimination of the wild-type and mutant alleles. Sanger sequencing $\mathrm{LOH}$ was considered when the intensity of any of the alleles was reduced relative to the other allele. For microsatellite $\mathrm{LOH}$, peak heights were measured and quantified as described below.

\section{LOH quantification}

The height in pixels of microsatellite peaks from the $\mathrm{LOH}$ study was measured with ImageJ. The unaltered $\mathrm{LOH}$ peak in each microsatellite was used as internal reference for comparison between normal and tumor samples. As an example, 167 pixels (normal) and 163 pixels (tumor) were used as internal reference in rs72225869. To calculate the ratio of $\mathrm{LOH}$, observed heights for the decreased peaks were divided by their respective references (rs72225869: normal 125/167 $=0.749$, tumor $97 / 163=0.595$; rs3034431: normal $175 / 128=1.367$, tumor $151 / 135=1.119$ ). Then, these normalized ratios were compared to obtain a final $\mathrm{LOH}$ ratio.

\section{Web resources}

1000 Genomes Project, https://www.internationalgenome.org/ EVS, https://evs.gs.washington.edu gnomAD, https://gnomad.broadinstitute.org PolyPhen, http://genetics.bwh.harvard.edu/pph2 SIFT, https://sift.bii.a-star.edu.sg MutationTaster, http://www.mutationtaster.org Human Splicing Finder (HSF), http://www.umd.be/HSF3 ImageJ, https://imagej.nih.gov/ij/index.html

\section{Results}

With the aim of finding germline colorectal cancer predisposition variants that would explain the increased cancer susceptibility in a cohort of colorectal cancer patients from FCCTX or high-risk MSS colorectal cancer families, the whole exome was studied by NGS in between 1 and 3 members of 18 families. In addition, an NGS panel of 94 cancer-related genes was used to study 62 patients from different families. After rigorous filtering, the NGS data showed three candidate variants in BRIP1: a frameshift variant and a missense variant identified by the whole-exome study and another frameshift variant detected by the multigene panel. Among the different candidate genes identified, BRIP1 was selected based on its recent association with colorectal cancer (35) and its involvement in familial ovarian cancer $(31,38)$. The present report focuses on these three germline BRIP1 variants identified in three unrelated families.

The families where the BRIP1 variants were detected were H463, CC350, and CC41, which fulfilled either the Amsterdam I (CC350) or Bethesda criteria (H463 and CC41; Fig. 1). In addition, they all presented MSS tumors with normal expression of all MMR proteins, and did not carry any germline mutation in MLH1, MSH2, MSH6, or PMS2 (Table 1). The three BRIP1 variants were validated by Sanger sequencing, and the segregation studies confirmed that the variants were present in all the affected members studied in each of the families (Fig. 1). Preliminary results for family H463 had been previously published (24).

\section{Family H463: BRIP1 c.1702_1703del, p.(Asn568TrpfsTer9)}

The BRIP1 variant identified by whole-exome sequencing in family $\mathrm{H} 463$ was a frameshift mutation known as NM 032043.2 c.1702_1703del, p.(Asn568TrpfsTer9), rs1057519365 (Table 1). This mutation consisted of a deletion of two adenines at positions 1702 and 1703 of the cDNA, located in exon 12 (Supplementary Fig. S1), and was found in family member II:6 (Fig. 1). This family was included in a Spanish collaborative study published in 2016 (24). Unfortunately, the segregation study could not be carried out due to the lack of availability of germline or tumor samples from any other members of the family. The $\mathrm{LOH}$ analysis showed that the tumor of member II: 6 presented a 
H463: NM_032043.2: c.1702_1703del, p.(Asn568TrpfsTer9)

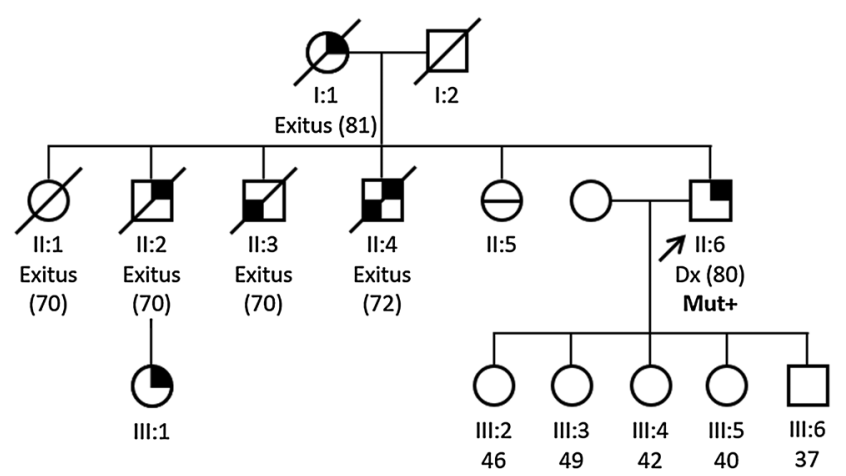

CRC, breast cancer

$\ominus$ Stomach cancer

$\square$ CRC

- Hodgkin lymphoma

Throat cancer

Bladder cancer

CC350: NM_032043.2: c.2220G>T, p.(GIn740His)

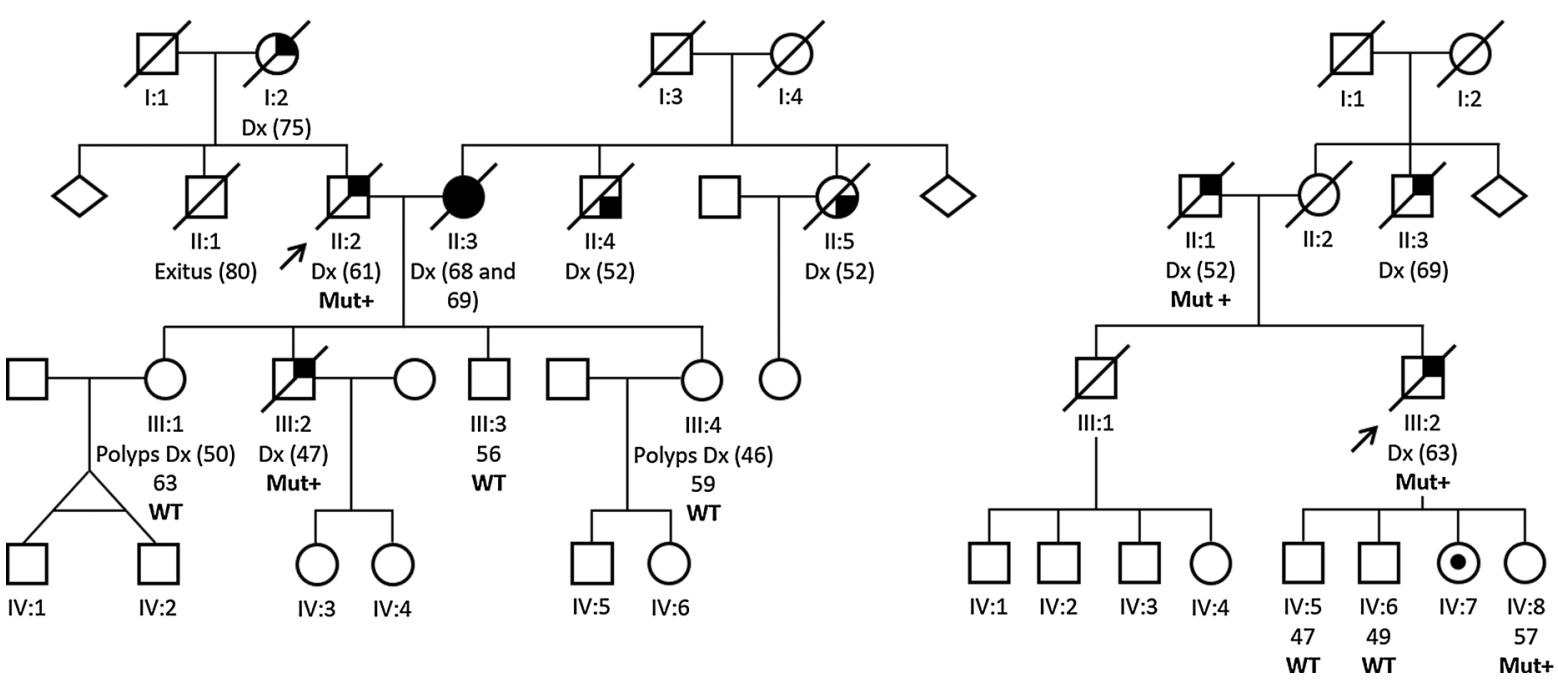

Figure 1.

Pedigrees of the three families in which the BRIPI variants were identified. Pedigrees of families $\mathrm{H} 463, \mathrm{C} 350$, and CC41. The symbol legend is shown in the top right corner. Ages at cancer diagnosis or at exitus (between brackets) and current ages (without brackets) are indicated when available below each individual. Family members in which the segregation of the corresponding BRIPI variant was studied are shown as either Mut+ (mutation carrier) or WT (wild-type). The arrows indicate the index case or proband of each family.

reduction of the wild-type allele compared with the mutant allele for both microsatellites studied. The $\mathrm{LOH}$ ratio observed was $20.5 \%$ for rs 72225869 and $18.1 \%$ for rs3034431 (Fig. 2). This deletion of two nucleotides in BRIP1 causes a shift of the reading frame of the codons, with the consequent change of an asparagine for a tryptophan at codon 568 , followed by a premature stop codon at position 9 of the new reading frame and the subsequent loss of normal protein function. On the other hand, this frameshift variant is located between the two helicase domains of the protein (Fig. 3), and its allele frequency in the general population is $1 / 251,398$ according to gnomAD. Furthermore, based on current information, the variant is considered as either pathogenic or likely pathogenic in ClinVar (6 submissions, May 2020).

\section{Family CC350: BRIP1 c.2220G >T, p.(GIn740His)}

The BRIP1 variant identified by whole-exome sequencing in family CC350 was a missense variant known as NM_032043.2
c.2220G > T, p.(Gln740His), rs45589637 (Table 1). This singlenucleotide variant consisted in the substitution of a guanine for a thymine at position of 2220 of the cDNA, located in exon 15 (Supplementary Fig. S2). The segregation study in the available family members showed that this variant segregates with colorectal cancer in the proband (II:2, diagnosed of colorectal cancer at 61) and his son (III:2, diagnosed of a rectal neoplasm with liver metastases at 47; Fig. 1). However, it was not carried by a healthy relative, nor by another two family members diagnosed with polyps (III:1 and III:4, with tubular adenomas of between 4 and $6 \mathrm{~mm}$ that presented mild or moderate dysplasia). It is worth noting that there was no evidence of a precursor adenoma in the pathologic specimens of the BRIP1 carriers who developed cancer. The $\mathrm{LOH}$ analysis in the tumor of member III: 2 showed that the difference between the wild-type and mutant alleles was minimal, and thus this study was considered as no evidence 
BRIP1 and Colorectal Cancer

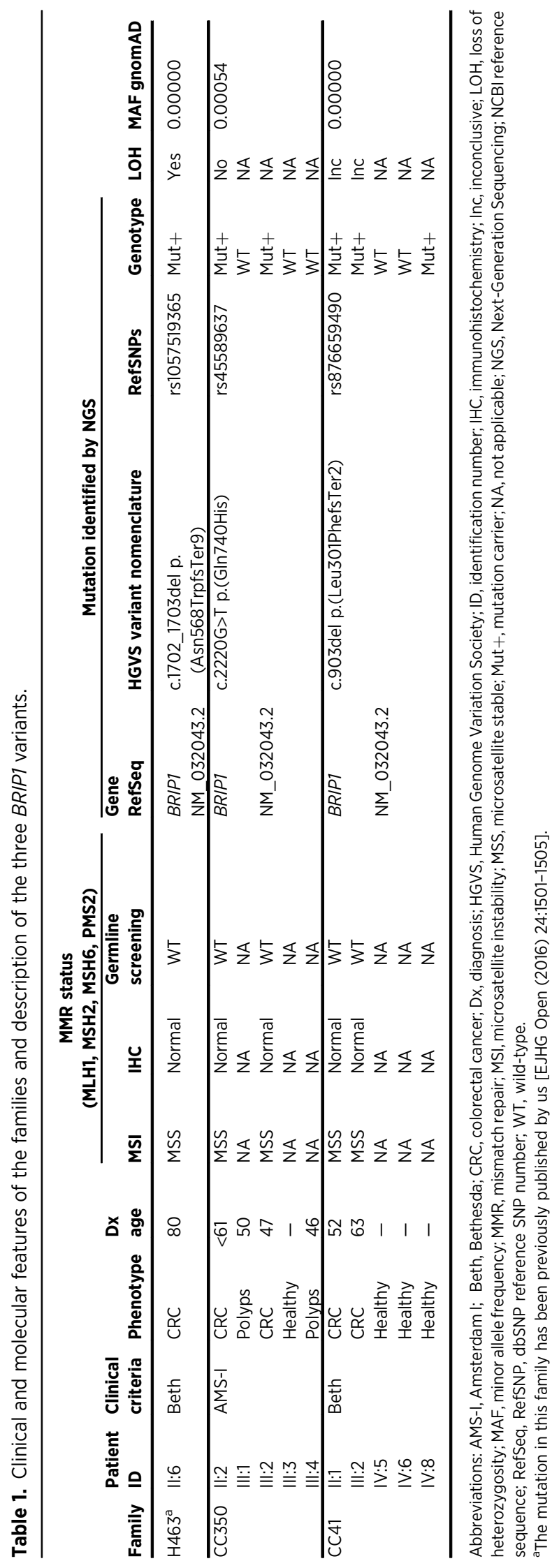


H463 family:

NM_032043.2: c.1702_1703delAA, p.(Asn568TrpfsTer9)

\section{CC350 family:} NM_032043.2: c.2220G>T, p.(Gln740His)

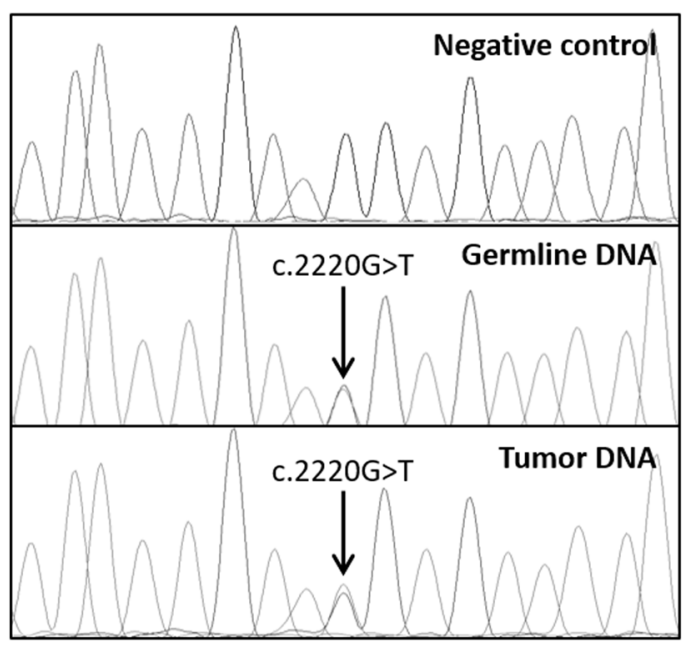

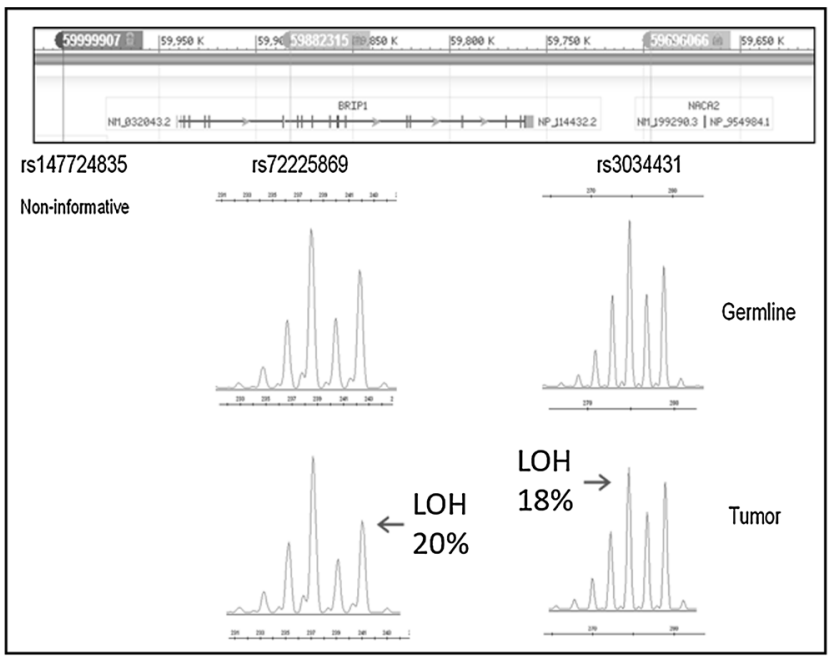

CC41 family: NM_032043.2: c.903del, p.Leu301PhefsTer2)

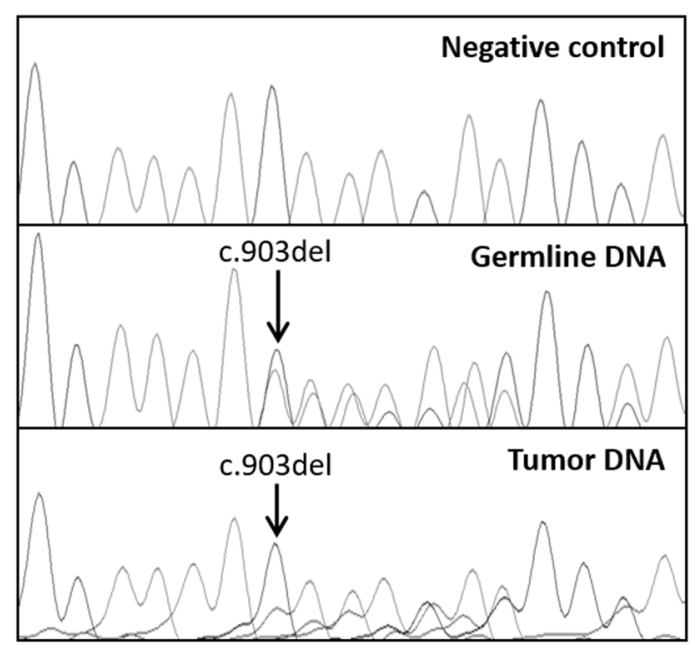

Figure 2.

LOH studies in the tumors of BRIP1 mutation carriers. The LOH was assessed by either the study of microsatellites (family H463) or Sanger sequencing (families CC350 and CC41). For family H463, the fragment analysis of microsatellites rs 72225869 and rs303443 in germline and tumor DNA from a carrier shows a decrease of the wild-type allele compared with the mutant allele. The LOH ratio was $20.5 \%$ for rs 72225869 and $18.1 \%$ for rs 3034431 . Conversely, there was no evidence of LOH in family CC350, whereas a partial decrease of the mutant allele was observed in the tumor of family CC41.

of LOH (Fig. 2). This variant is located in the Helicase Cterminal (HELICc) domain (Fig. 3) and has an allele frequency in the general population of 0.00041 according to gnomAD. In silico studies considered this variant as "Deleterious" by SIFT, "Possibly Damaging" by PolyPhen-2, and "Disease Causing" by MutationTaster. However, this singlenucleotide variant is classified as either a variant of uncertain significance (VUS; 10 submissions) or likely benign (5 submissions) by ClinVar (May 2020).

\section{Family CC41: BRIP1 c.903del, p.(Leu301PhefsTer2)}

The BRIP1 variant detected by the multigene panel (39) in family CC41 was a frameshift mutation known as NM_032043.2 c.903del, p.(Leu301PhefsTer2), rs876659490 (Table 1). This mutation consisted on the deletion of a guanine at position 903 of the cDNA, with a consequent shift of the reading frame of the codons (Supplementary Fig. S3). The segregation study in the available family members showed that this variant segregates with colorectal cancer in the proband (III:2) and his father (II:1), both affected with colorectal cancer at ages 63 and 52, respectively. The variant was also carried by his 57 -year-old healthy daughter (IV:8), whereas another two healthy sons of ages 47 (IV:5) and 49 (IV:6) were noncarriers (Fig. 1). The LOH analysis of this mutation revealed that the tumor from member II:1 showed some decrease of the mutant allele and increase of the wild-type 


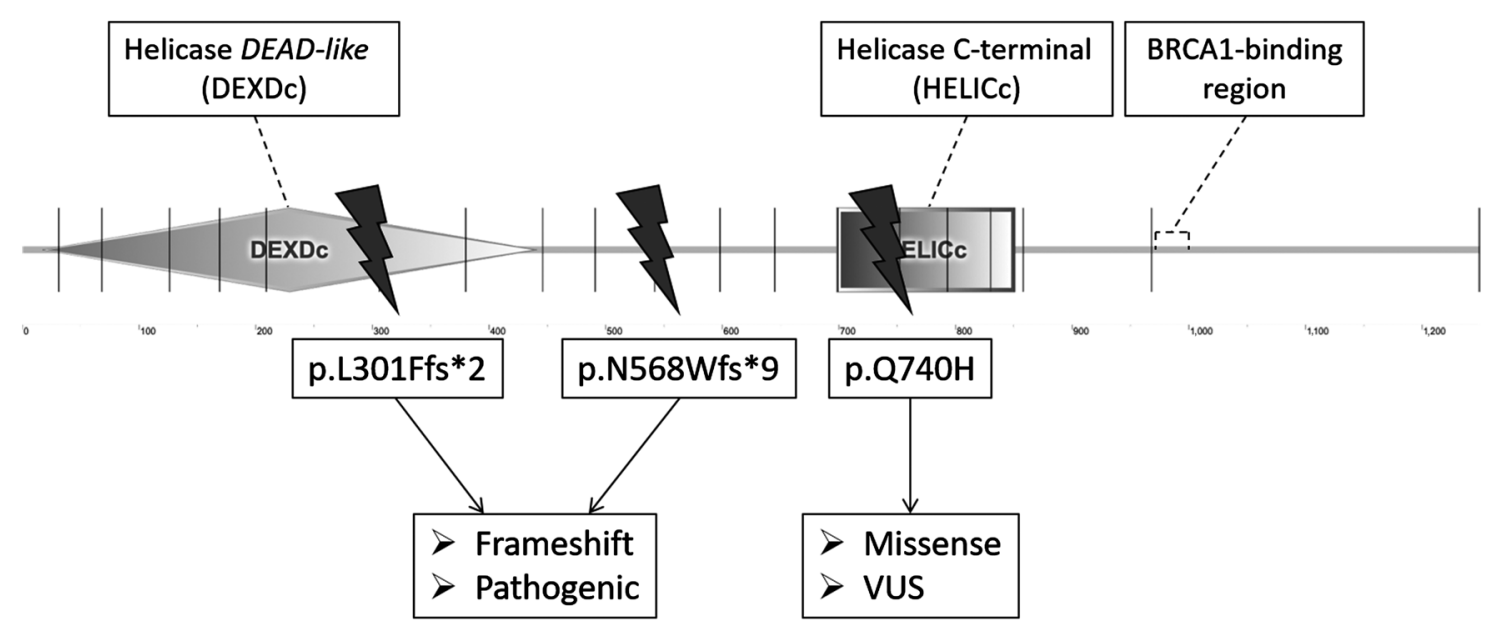

Figure 3.

Functional domains of the BRIP1 protein and location of the variants. Representation of BRIP1's functional domains and BRCA1-binding region (amino acids 979-1006; Cantor et al., ref. 40). The lightning bolts indicate the location of the identified variants within the protein and the nomenclature of the variants at protein level is shown in the corresponding boxes underneath them.

allele (Fig. 2). This variant is located in the Helicase DEADlike (DEXDc) domain (Fig. 3) and is a novel variant with no frequency data in the general population. Furthermore, it is a frameshift variant whose consequence is a nonfunctional truncated protein. The variant has been reported in ClinVar as pathogenic (1 submission, May 2020) and the segregation in the family supports its pathogenicity.

\section{Discussion}

FCCTX encompasses a group of families that fulfill the Amsterdam criteria for HNPCC but with no defects in the MMR genes and a still unknown genetic basis. The identification of pathogenic mutations in new genes involved in the cancer inheritance of these families can provide valuable information to be used during patient screening and genetic counseling. In addition, a better understanding of hereditary colorectal cancer may provide important clues to disease predisposition that could contribute to molecular diagnostics, improved risk stratification, and targeted therapeutic strategies. Given a recently reported association between BRIP1 and colorectal cancer (35) and its well-known effect on ovarian cancer risk $(31,38)$, the aim of the present study was to evaluate the role of BRIP1 variants and their association with inherited colorectal cancer.

Here, we present data from three unrelated families where we detected three different BRIP1 variants. Two of the variants cause a translational shift of the reading frame, resulting in the premature truncation of the protein, the loss of at least one of the two helicase domains and the BRCA1-binding region (40), and the expected loss of function of the protein. Therefore, they are both classified as pathogenic and have been indeed reported as pathogenic in ClinVar. The segregation study of variant BRIP1 c.903del, p.(Leu301PhefsTer2) in family CC41 is consistent with its pathogenicity. The LOH study of BRIP1 c.1702_1703del, p.(Asn568TrpfsTer9) in family H463 suggests the loss of the wild-type allele, which could be a possible second hit pointing to its potential implication in cancer development. However, the LOH study in family CC41 is not informative. Altogether, these results are consistent with the pathogenicity of both frameshift variants and support the involvement of $B R I P 1$ in the colorectal cancer inheritance of the corresponding families.

On the other hand, the missense variant carried by family CC350 is predicted to be deleterious by in silico tools and has been reported in individuals affected with ovarian cancer (31), peritoneal cancer (41), breast cancer $(30,33,42)$, and in individuals undergoing testing for Lynch syndrome (43). However, its ClinVar entry classifies it as either benign or of uncertain significance. This variant seems to segregate with colorectal cancer in family CC350. It is worth noting that CC350 family member III:2 has a bilinear family history of cancer, given that in the maternal side his mother was diagnosed of breast cancer at the age of 69 and colorectal cancer at 88 , and two of his uncles were diagnosed of throat cancer at 52. However, given that his father and paternal grandfather were diagnosed of colorectal cancer at 61 and 75, respectively, it is more likely that the colorectal cancer heritability comes by this side. The LOH study showed no evidence of LOH. Taking all this into account, we cannot confirm the pathogenicity of this variant until functional studies have been performed, because the available evidence is currently insufficient to determine the role of this variant in the disease. For that reason, it still remains as a VUS and patient recommendations should be based on the individual's personal and family history instead of on the variant. Longer follow-up of noncarrier members is also needed to see if it is associated with colorectal cancer.

$B R I P 1$ encodes a member of the RecQ DEAH helicase family that interacts with $B R C A 1$ and plays an important role in $B R C A 1^{\prime}$ s normal double-strand break repair function. Biallelic 
pathogenic variants in BRIP1 are known to cause FA type J, but this gene is also a target of germline cancer-inducing mutations. In fact, monoallelic pathogenic germline variants in BRIP1 are reported to confer up to $10 \%$ cumulative risk for ovarian cancer $(31,32)$, whereas they may or may not be involved in breast cancer risk $(44,45)$. On the other hand, BRIP1 variants have been reported in $3 \%$ of colorectal cancer samples according to The Cancer Genome Atlas, but their association with colorectal cancer is poorly documented. Thanks to a previous collaborative study, we identified the first BRIP1 germline variant in a colorectal cancer family (19), but more recently the association between BRIP1 and colorectal cancer has been confirmed by additional studies that have reported pathogenic BRIP1 germline variants in colorectal cancer cases with a strong family history (35). Rosenthal and colleagues reported a significant enrichment of potentially disruptive BRIP1 variants in colorectal cancer and polyposis cases (36). The authors suggest that BRIP1 may be associated with a colorectal cancer syndrome possibly at lower penetrance than with ovarian cancer, and support further investigating the association between BRIP1 and colorectal cancer risk (36). A heterozygous missense BRIP1 candidate variant was also identified by Thutkawkorapin and colleagues in an early-onset colorectal cancer cohort, although further studies are needed to confirm its pathogenicity (37). In addition, more recently a case report by Ali and colleagues described two colorectal cancer patients with a strong family history who presented BRIP1 mutations in tumor tissue that were later shown to be carried in the germline, postulating that germline BRIP1 mutations confer an increased risk of developing colorectal cancer (35). In line with these studies, our results suggest that the two germline BRIP1 truncating variants identified in our cohort are involved in the colorectal cancer development of the carrier families, whereas the BRIP1 missense variant is a candidate variant whose pathogenicity is still to be determined. The main limitations of the present work are the restricted number of families studied and the difficulty of recruiting additional family members in order to have informative segregation studies.

\section{References}

1. Bray F, Ferlay J, Laversanne M, Brewster DH, Gombe Mbalawa C, Kohler B, et al. Cancer incidence in five continents: inclusion criteria, highlights from volume $\mathrm{X}$ and the global status of cancer registration. Int J Cancer 2015;137:2060-71.

2. Arnold M, Sierra MS, Laversanne M, Soerjomataram I, Jemal A, Bray F. Global patterns and trends in colorectal cancer incidence and mortality. Gut 2017;66:683-91.

3. Grady WM. Genetic testing for high-risk colon cancer patients. Gastroenterology 2003;124:1574-94.

4. Lichtenstein P, Holm NV, Verkasalo PK, Iliadou A, Kaprio J, Koskenvuo M, et al. Environmental and heritable factors in the causation of cancer-analyses of cohorts of twins from Sweden, Denmark, and Finland. N Engl J Med 2000;343:78-85.
In conclusion, we suggest that BRIP1 pathogenic germline variants may have a causal role in colorectal cancer as moderate-penetrance cancer susceptibility alleles and be associated with hereditary colorectal cancer predisposition. To further prove this, BRIP1 should be included in clinical panels for the evaluation of germline mutations in hereditary cancer susceptibility genes, and larger studies should be done to provide more evidence of this association.

\section{Supplementary Data}

Supplementary data include a table with the primer sequences and three figures showing the validation and segregation studies of the families carrying the $B R I P 1$ variants.

\section{Authors' Disclosures}

Vanesa García-Barberan reports grants from AstraZeneca and personal fees from Amgen outside the submitted work. No disclosures were reported by the other authors.

\section{Authors' Contributions}

L. Martin-Morales: Supervision, investigation. P. Garre: Supervision. V. Lorca: Software. M. Cazorla: Validation. P. Llovet: Visualization. I. Bando: Methodology. Vanesa García-Barberan: Supervision. M.L. Gonzalez-Morales: Formal analysis. C. Esteban-Jurado: Methodology. M. de la Hoya: Supervision. S. Castellví-Bel: Validation. T. Caldes: Funding acquisition, writing-original draft, writing-review, and editing.

\section{Acknowledgments}

The authors would like to thank the families for taking part in the study. We would also like to acknowledge Laia Bonjoch for the data of BRIP1 LOH quantification in family H463, Paula Diaque and Isabel Díaz Millán for their technical assistance, and the Biobank of Hospital Clínico San Carlos for the FFPE blocks, sections and pathology records. This work was supported by grants from Fondo de Investigación Sanitaria/FEDER PI16/01292 and RTC-2016-5092-1 (Instituto de Salud Carlos III, Ministerio de Ciencia, Innovación y Universidades).

The costs of publication of this article were defrayed in part by the payment of page charges. This article must therefore be hereby marked advertisement in accordance with 18 U.S.C. Section 1734 solely to indicate this fact.

Received June 16, 2020; revised August 17, 2020; accepted October 15, 2020; published first October 28, 2020.

5. Stoffel EM, Kastrinos F. Familial colorectal cancer, beyond Lynch syndrome. Clin Gastroenterol Hepatol 2014;12:1059-68.

6. Watson P, Lynch HT. Extracolonic cancer in hereditary nonpolyposis colorectal cancer. Cancer 1993;71:677-85.

7. Vasen HF, Mecklin JP, Khan PM, Lynch HT. The international collaborative group on hereditary non-polyposis colorectal cancer (ICG-HNPCC). Dis Colon Rectum 1991;34:424-5.

8. Vasen HF, Watson P, Mecklin JP, Lynch HT. New clinical criteria for hereditary nonpolyposis colorectal cancer (HNPCC, Lynch syndrome) proposed by the International Collaborative group on HNPCC. Gastroenterology 1999;116:1453-6.

9. Lynch HT, Riegert-Johnson DL, Snyder C, Lynch JF, Hagenkord J, Boland CR, et al. Lynch syndrome-associated extracolonic tumors are 
rare in two extended families with the same EPCAM deletion. Am J Gastroenterol 2011;106:1829-36.

10. Lynch HT, Lynch PM, Lanspa SJ, Snyder CL, Lynch JF, Boland CR. Review of the Lynch syndrome: history, molecular genetics, screening, differential diagnosis, and medicolegal ramifications. Clin Genet 2009; 76:1-18.

11. Rodriguez-Bigas MA, Boland CR, Hamilton SR, Henson DE, Jass JR, Khan PM, et al. A national cancer institute workshop on hereditary nonpolyposis colorectal cancer syndrome: meeting highlights and Bethesda guidelines. J Natl Cancer Inst 1997;89:1758-62.

12. Lindor NM, Rabe K, Petersen GM, Haile R, Casey G, Baron J, et al. Lower cancer incidence in Amsterdam-I criteria families without mismatch repair deficiency: familial colorectal cancer type X. JAMA 2005;293:1979-85.

13. Sánchez-de-Abajo A, de la Hoya $M$, van Puijenbroek M, Tosar $A$, López-Asenjo JA, Díaz-Rubio E, et al. Molecular analysis of colorectal cancer tumors from patients with mismatch repair proficient hereditary nonpolyposis colorectal cancer suggests novel carcinogenic pathways. Clin Cancer Res 2007;13:5729-35.

14. Garre P, Martín L, Bando I, Tosar A, Llovet P, Sanz J, et al. Cancer risk and overall survival in mismatch repair proficient hereditary nonpolyposis colorectal cancer, Lynch syndrome and sporadic colorectal cancer. Fam Cancer 2014;13:109-19.

15. Dominguez-Valentin M, Therkildsen C, Da Silva S, Nilbert M. Familial colorectal cancer type X: genetic profiles and phenotypic features. Mod Pathol 2015;28:30-6.

16. Garre P, Briceño V, Xicola RM, Doyle BJ, de la Hoya M, Sanz J, et al. Analysis of the oxidative damage repair genes NUDT1, OGG1, and MUTYH in patients from mismatch repair proficient HNPCC families (MSS-HNPCC). Clin Cancer Res 2011;17:1701-12.

17. Garre P, Martín L, Sanz J, Romero A, Tosar A, Bando I, et al. BRCA2 gene: a candidate for clinical testing in familial colorectal cancer type X. Clin Genet 2015;87:582-7.

18. Palles C, Cazier J-B, Howarth KM, Domingo E, Jones AM, Broderick P, et al. Germline mutations affecting the proofreading domains of POLE and POLD1 predispose to colorectal adenomas and carcinomas. Nat Genet 2013;45:136-44.

19. Esteban-Jurado C, Vila-Casadesús M, Garre P, Lozano JJ, Pristoupilova A, Beltran S, et al. Whole-exome sequencing identifies rare pathogenic variants in new predisposition genes for familial colorectal cancer. Genet Med 2015;17:131-42.

20. Seguí N, Mina LB, Lázaro C, Sanz-Pamplona R, Pons T, Navarro M, et al. Germline mutations in FAN1 cause hereditary colorectal cancer by impairing DNA repair. Gastroenterology 2015;149:563-6.

21. Weren RDA, Ligtenberg MJL, Kets CM, de Voer RM, Verwiel ETP, Spruijt L, et al. A germline homozygous mutation in the base-excision repair gene NTHL1 causes adenomatous polyposis and colorectal cancer. Nat Genet 2015;47:668-71.

22. Martín-Morales L, Feldman M, Vershinin Z, Garre P, Caldés T, Levy D. SETD6 dominant negative mutation in familial colorectal cancer type X. Hum Mol Genet 2017;26:4481-93.

23. Bellido F, Sowada N, Mur P, Lázaro C, Pons T, Valdés-Mas R, et al. Association between germline mutations in BRF1, a subunit of the RNA polymerase III transcription complex, and hereditary colorectal cancer. Gastroenterology 2018;154:181-94.

24. The EPICOLON Consortium, Esteban-Jurado C, Franch-Expósito S, Muñoz J, Ocaña T, Carballal S, et al. The Fanconi anemia DNA damage repair pathway in the spotlight for germline predisposition to colorectal cancer. Eur J Hum Genet 2016;24:1501-5.

25. Wang W. Emergence of a DNA-damage response network consisting of Fanconi anaemia and BRCA proteins. Nat Rev Genet 2007;8:735-48.

26. Chen X, Wilson JB, McChesney P, Williams SA, Kwon Y, Longerich S, et al. The Fanconi anemia proteins FANCD2 and FANCJ interact and regulate each other's chromatin localization. J Biol Chem 2014;289: 25774-82.

27. Ali AM, Singh TR, Meetei AR. FANCM-FAAP24 and FANCJ: FA proteins that metabolize DNA. Mutat Res 2009;668:20-6.

28. Levitus M, Waisfisz Q, Godthelp BC, Vries Y de, Hussain S, Wiegant WW, et al. The DNA helicase BRIP1 is defective in Fanconi anemia complementation group J. Nat Genet 2005;37:934-5.

29. Levran O, Attwooll C, Henry RT, Milton KL, Neveling K, Rio P, et al. The BRCA1-interacting helicase BRIP1 is deficient in Fanconi anemia. Nat Genet 2005;37:931-3.

30. Litman R, Peng M, Jin Z, Zhang F, Zhang J, Powell S, et al. BACH1 is critical for homologous recombination and appears to be the Fanconi anemia gene product FANCJ. Cancer Cell 2005;8:255-65.

31. Ramus SJ, Song H, Dicks E, Tyrer JP, Rosenthal AN, Intermaggio MP, et al. Germline mutations in the BRIP1, BARD1, PALB2, and NBN genes in women with ovarian cancer. Natl Cancer Inst 2015; 107:djv214.

32. Rafnar T, Gudbjartsson DF, Sulem P, Jonasdottir A, Sigurdsson A, Jonasdottir A, et al. Mutations in BRIP1 confer high risk of ovarian cancer. Nat Genet 2011;43:1104-7.

33. Easton DF, Lesueur F, Decker B, Michailidou K, Li J, Allen J, et al. No evidence that protein truncating variants in BRIP1 are associated with breast cancer risk: implications for gene panel testing. J Med Genet 2016;53:298-309.

34. Couch FJ, Shimelis H, Hu C, Hart SN, Polley EC, Na J, et al. Associations between cancer predisposition testing panel genes and breast cancer. JAMA Oncol 2017;3:1190-6.

35. Ali M, Delozier CD, Chaudhary U. BRIP-1 germline mutation and its role in colon cancer: presentation of two case reports and review of literature. BMC Med Genet 2019;20:75.

36. Rosenthal EA, Shirts BH, Amendola LM, Horike-Pyne M, Robertson $\mathrm{PD}$, Hisama FM, et al. Rare loss of function variants in candidate genes and risk of colorectal cancer. Hum Genet 2018;137:795-806.

37. Thutkawkorapin J, Lindblom A, Tham E. Exome sequencing in 51 early onset non-familial CRC cases. Mol Genet Genomic Med 2019;7: e605.

38. Suszynska M, Ratajska M, Kozlowski P. BRIP1, RAD51C, and RAD51D mutations are associated with high susceptibility to ovarian cancer: mutation prevalence and precise risk estimates based on a pooled analysis of $\sim 30,000$ cases. J Ovarian Res 2020;13:50.

39. Martin-Morales L, Rofes P, Diaz-Rubio E, Llovet P, Lorca V, Bando I, et al. Novel genetic mutations detected by multigene panel are associated with hereditary colorectal cancer predisposition. PLoS One 2018;13:e203885.

40. Cantor S, Drapkin R, Zhang F, Lin Y, Han J, Pamidi S, et al. The BRCA1-associated protein BACH1 is a DNA helicase targeted by clinically relevant inactivating mutations. Proc Natl Acad Sci 2004; 101:2357-62.

41. Kahn R, Garcia-Soto A, Silva-Smith R, Pinto A, George SHL. Primary peritoneal carcinoma in a BRCA1/2-negative, PALB2-positive patient. Gynecol Oncol Rep 2016;17:93-5.

42. Jalkh N, Chouery E, Haidar Z, Khater C, Atallah D, Ali H, et al. Nextgeneration sequencing in familial breast cancer patients from Lebanon. BMC Med Genomics 2017;10:8.

43. Yurgelun MB, Allen B, Kaldate RR, Bowles KR, Judkins T, Kaushik P, et al. Identification of a variety of mutations in cancer predisposition genes in patients with suspected lynch syndrome. Gastroenterology 2015;149:604-613.

44. Li J, Meeks H, Feng B-J, Healey S, Thorne H, Makunin I, et al. Targeted massively parallel sequencing of a panel of putative breast cancer susceptibility genes in a large cohort of multiple-case breast and ovarian cancer families. J Med Genet 2016;53:34-42.

45. Shuen AY, Foulkes WD. Inherited mutations in breast cancer genesrisk and response. J Mammary Gland Biol Neoplasia 2011;16:3-15. 

Research. 


\section{Cancer Prevention Research}

\section{BRIP1, a Gene Potentially Implicated in Familial Colorectal Cancer Type X}

Lorena Martín-Morales, Pilar Garre, Víctor Lorca, et al.

Cancer Prev Res 2021;14:185-194. Published OnlineFirst October 28, 2020.

\section{Updated version Access the most recent version of this article at: doi:10.1158/1940-6207.CAPR-20-0316}

Supplementary Access the most recent supplemental material at:

Material http://cancerpreventionresearch.aacrjournals.org/content/suppl/2020/10/28/1940-6207.CAPR-20-0316.DC 1

Cited articles This article cites 45 articles, 7 of which you can access for free at:

http://cancerpreventionresearch.aacrjournals.org/content/14/2/185.full\#ref-list-1

E-mail alerts Sign up to receive free email-alerts related to this article or journal.

Reprints and

To order reprints of this article or to subscribe to the journal, contact the AACR Publications Department at Subscriptions pubs@aacr.org.

Permissions To request permission to re-use all or part of this article, use this link http://cancerpreventionresearch.aacrjournals.org/content/14/2/185.

Click on "Request Permissions" which will take you to the Copyright Clearance Center's (CCC) Rightslink site. 